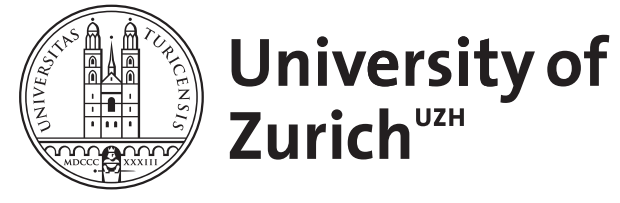

Zurich Open Repository and Archive

University of Zurich

University Library

Strickhofstrasse 39

CH-8057 Zurich

www.zora.uzh.ch

Year: 2011

\title{
Rechtliche Aspekte personalisierter Medizin
}

Tag, Brigitte

Posted at the Zurich Open Repository and Archive, University of Zurich

ZORA URL: https://doi.org/10.5167/uzh-51719

Journal Article

Published Version

Originally published at:

Tag, Brigitte (2011). Rechtliche Aspekte personalisierter Medizin. Schweizerische Ärztezeitung, 92(32/33):1207. 


\title{
Rechtliche Aspekte personalisierter Medizin
}

\author{
Bald wird das gesamte menschliche Genom leicht verfügbar sein. Dies führt zu einem \\ Paradigmenwechsel in der medizinischen Forschung und dem Gesundheitswesen. \\ Die Aussicht darauf fordert nicht zuletzt die Gesetzgebung jedes Landes.
}

Brigitte Tag

Professorin für Strafrecht, Strafverfahrensrecht und Medizinrecht,

Universität Zürich

Die personalisierte Medizin steht für eine das Gesundheitswesen nachhaltig verändernde Entwicklung. Zwar entspricht es bereits heute dem Berufsverständnis von Ärztinnen und Ärzten, den Patienten mittels einer auf seine Krankheit individuell zugeschnittenen Therapie zu behandeln. Trotzdem erweist sich die Differentialdiagnose nicht immer als zutreffend, schlägt beim konkreten Patienten die gewählte Therapie nicht oder nur suboptimal an.

Die personalisierte Medizin mit ihrer Fokussierung auf die molekularen Ursachenzusammenhänge des Krankheitsgeschehens soll hier weiterhelfen. Eine Vision ist, dass das Personal-Genom-Project, als Weiterentwicklung des Human-Genom-Projects, die Daten von Einzelpersonen decodiert, speichert und für medizinische Forschung sowie Behandlung zur Verfügung stellt. Trotz der damit verbundenen offenen rechtlichen und ethischen Fragen wird dadurch die personalisierte Medizin vorangebracht. Die Hürden,

\section{Für personalisierte Medizin müssen neben Biomarkern auch angemessene Lösungen für ethische und rechtliche Fragen entwickelt werden.}

Korrespondenz:

Prof. Dr. iur. utr. Brigitte Tag Lehrstuhl für Strafrecht,

Strafprozessrecht und

Medizinrecht

Universität Zürich

Rechtswissenschaftliches Institut

Freiestrasse 15

CH-8032 Zürich

Tel. 0446343939

Fax 0446344937

brigitte.tag@rwi.uzh.ch die es zu nehmen gilt, sind jedoch hoch: Die im Bundesgesetz über die Krankenversicherung KVG verankerten WZW-Kriterien (Wirksamkeit, Zweckmässigkeit, Wirtschaftlichkeit) verlangen den Nachweis der Wirksamkeit einer medizinischen Behandlung, sollen die damit zusammenhängenden Kosten von der Grundversicherung übernommen werden. Auch der Arzt orientiert sich so weit möglich an Standards und Leitlinien, andernfalls setzt er sich schneller dem Behandlungsfehlervorwurf und den damit gekoppelten Haftungsfragen aus. Denn der Nachweis der (potentiellen) Wirksamkeit einer lege artis durchgeführten Therapie wird in der Regel statistisch, d.h. an grösseren Patientengruppen geführt. Dies gilt unabhängig davon, dass einzelne Patienten bei gleicher Diagnose unterschiedlich auf die zur Verfügung gestellten Therapiekonzepte reagieren. Liegen die Ursachen hierfür auf der molekularen Ebene bzw. im Zusammenspiel von genetischer Disposition und den persönlichen Lebensumständen des Patienten, können Biomarker
Patientengruppen jedoch klinisch relevanten Subgruppen zuweisen. Damit eröffnen sich zielgenauere Diagnosen und Therapien. Doch auch mit dieser Stratifizierung sind grosse Herausforderungen verbunden.

Die Identifizierung neuer Biomarker ist komplex, beruht auf der Erhebung, Verarbeitung und Interpretation extrem grosser Datenmengen. Sie stellt hohe Anforderungen an die technische Machbarkeit, die fachliche Interpretation, die Finanzierung, die Belastbarkeit des Patienten, der Ärzte und des Gesundheitswesens insgesamt. Das bedeutet auch, dass zu diskutieren ist, ob und wie weit personalisierte Medizin vom KVG getragen werden kann und soll. Zudem steigert die Verbindung von personalisierter und informationsbasierter Medizin nicht nur die Qualität der Gesundheitsversorgung, sondern verstärkt auch das Risiko des Datenmissbrauchs. Dass die erhobenen Daten gerade auch der Forschung dienen sollen, führt zu weiteren zentralen Fragen wie zur Reichweite der Einwilligung, zum individuellen und gesellschaftlichen Nutzen und dessen Grenzen. Die Diskussion um personalisierte Medizin wird die Suche nach den Antworten unterstützen - und dazu beitragen, neben den Biomarkern auch angemessene Lösungen für die ethischen und rechtlichen Fragen zu entwickeln.

\section{Symposium «Personalisierte Medizin» in Engelberg/OW}

Der «10th Dialogue on Science» in Engelberg vom 14. bis 16. September 2011 bietet eine einmalige Plattform, um sich über individualisierte Medizin, ihr Potential, die Möglichkeiten und Herausforderungen zu informieren und darüber zu diskutieren. Führende Experten aus Wissenschaft, Gesundheitswesen, privaten GentestFirmen, Ethik und Politik werden sich am Wissenschaftskongress mit den Teilnehmenden und jungen Wissenschaftlern über die kontroversen Folgen dieser Technologie für unsere Gesellschaft austauschen. Weitere Informationen: www.academia-engelberg.ch $\rightarrow$ Konferenzen $\rightarrow$ Konferenz 2011.

Die Schweizerische Ärztezeitung ist Medienpartner des Symposiums. 